\title{
The differentiation of fibre- and drug type Cannabis seedlings by gas chromatography/mass spectrometry and chemometric tools
}

\author{
Julian Broséus, Frédéric Anglada ${ }^{1}$, Pierre Esseiva * \\ Institut de Police Scientifique, School of Criminal Sciences, Batochime, University of Lausanne, 1015 Lausanne-Dorigny, Switzerland
}

\section{A R T I C L E I N F O}

\section{Article history:}

Received 11 December 2009

Received in revised form 1 March 2010

Accepted 20 March 2010

Available online 24 April 2010

\section{Keywords:}

Cannabis

Cannabis chemotype

Gas chromatography-mass spectrometry

Support vector machines

\begin{abstract}
A B S T R A C T
Cannabis cultivation in order to produce drugs is forbidden in Switzerland. Thus, law enforcement authorities regularly ask forensic laboratories to determinate cannabis plant's chemotype from seized material in order to ascertain that the plantation is legal or not. As required by the EU official analysis protocol the THC rate of cannabis is measured from the flowers at maturity. When laboratories are confronted to seedlings, they have to lead the plant to maturity, meaning a time consuming and costly procedure. This study investigated the discrimination of fibre type from drug type Cannabis seedlings by analysing the compounds found in their leaves and using chemometrics tools. 11 legal varieties allowed by the Swiss Federal Office for Agriculture and 13 illegal ones were greenhouse grown and analysed using a gas chromatograph interfaced with a mass spectrometer. Compounds that show high discrimination capabilities in the seedlings have been identified and a support vector machines (SVMs) analysis was used to classify the cannabis samples. The overall set of samples shows a classification rate above $99 \%$ with false positive rates less than $2 \%$. This model allows then discrimination between fibre and drug type Cannabis at an early stage of growth. Therefore it is not necessary to wait plants' maturity to quantify their amount of THC in order to determine their chemotype. This procedure could be used for the control of legal (fibre type) and illegal (drug type) Cannabis production.
\end{abstract}

(c) 2010 Elsevier Ireland Ltd. All rights reserved.

\section{Introduction}

More than 400 chemical compounds have been isolated in Cannabis sativa L. of which more than 60 are cannabinoids. Cannabinoids are a characteristic class of substances unique to Cannabis. The most abundant are $\Delta^{9}$-tetrahydrocannabinol $\left(\Delta^{9}\right.$ THC or THC, the main psychoactive cannabinoid), cannabidiol (CBD), cannabigerol (CBG) and cannabichromene (CBC). In fresh plant material, most of cannabinoids exist in the form of their acids precursors. For example, in fresh plant material, 95\% of THC and CBD consist of their precursors THCA-A and CBDA, respectively [1]. They are converted (by a decarboxylation) to their corresponding neutral cannabinoids in course of time, upon heating or under alkaline conditions. In this study we perform this decarboxylation process directly in the injector (in the liner) of the GC-MS [2] used for the analysis of the samples. In addition to cannabinoids, many constituents commonly encountered in nature like terpenes, alkanes, flavonoids and nitrogenous compounds are also present $[3,4]$.

\footnotetext{
* Corresponding author. Tel.: +412169246 52; fax: +41216924605. E-mail address: pierre.esseiva@unil.ch (P. Esseiva).

${ }^{1}$ We dedicate this article to the memory of our friend Frédéric Anglada, who unfortunately passed away on the 1st January 2010.
}

Cannabis sativa subspecies are divided into several chemical phenotypes. The relative proportions of THC, CBN and CBD have been used by various authors for distinguishing three predominant chemotypes; chemotypes I, II and III. Small and Beckstead [5] use the proportion of THC and CBD for classifying cannabis samples. For example, the main cannabinoid of the fibre type is CBD and the one of the drug type is THC. The following table summarizes the criteria used to discriminate chemotypes (Table 1 ).

The United Nations Office on Drugs and Crime [6] proposes to use a combination of three cannabinoids in the following index: $X=\frac{[\mathrm{THC}]+[\mathrm{CBN}]}{[\mathrm{CBD}]}$

with $[\mathrm{THC}],[\mathrm{CBN}]$ and $[\mathrm{CBD}]$ being the relative proportion (peak area) of respectively THC, CBN and CBD in the chromatogram. Cannabinol (CBN) is not a natural cannabinoid, it is a degradation product of THC. If this ratio is higher than 1 , the plant is classified as drug type cannabis or as chemotype I, while if index is lower than 1 , the plant is classified as fibre type cannabis or as chemotype III. A study performed in Greece [7] highlighted the difficulty for differentiating drug from fibre type chemotypes with an error rate of classification of about $20 \%$ depending on index used.

This chemotype classification is mainly carried out with mature cannabis and few researches have been focused on the possibility to attribute a chemotype using cannabis seedlings. Previous 
Table 1

Predominant Cannabis chemotypes according to THC and CBD content [5].

\begin{tabular}{|c|c|c|c|}
\hline Chemotype & Designation products & Predominant cannabinoids & THC to CBD ratio \\
\hline I: Drug type & $\begin{array}{l}\text { Illegal hemp, resinous } \\
\text { Cannabis } \\
\text { Marijuana, hashish }\end{array}$ & THC & $\begin{array}{l}\text { High ratio } \\
\text { THC content }>0.30 \%^{\mathrm{a}} \\
\text { CBD content }<0.50 \%^{\mathrm{a}}\end{array}$ \\
\hline II: Intermediate type & & THC, CBD & Ratio near $1(0.5-2)$ \\
\hline III: Fibre type & $\begin{array}{l}\text { Legal hemp, industrial } \\
\text { (textile) cannabis, Edible seed oil, essential oils, } \\
\text { cosmetics, lubricants, fuels, fibres }\end{array}$ & CBD & $\begin{array}{l}\text { Low ratio } \\
\text { THC }<0.30 \%\end{array}$ \\
\hline
\end{tabular}

a Dry weight of the reproductive part of the plant at flowering.

studies showed that the chemotype does not change in plants at different ages or in different sexes throughout the entire life cycle until flowering (even if the cannabinoids quantities are not constant). In fact, THC in chemotype I and CBD in chemotype III plants become the predominant cannabinoids very early (since 1 month). According to cannabinoids amounts and their ratio, it's possible to analyse Cannabis leaves at a very early phase of growth and to classify their chemotype $[8,9]$.

Cannabis cultivation in order to produce illicit drugs is forbidden in several countries, of which Switzerland. However, fibre type cannabis cultivation is legal in some countries, under restrictions. For example, European Union requires that seeds intended for sowing of fibre cannabis varieties show in the mature plant a THC amount lower than $0.2 \%(\mathrm{w} / \mathrm{w})[10]$ while Switzerland requires a THC amount lower than $0.3 \%(w / w)$ [11]. Thus, law enforcement authorities regularly ask forensic laboratories to determinate cannabis plant's chemotype from seized material in order to ascertain if the plantation is legal or not.

We propose then to develop a GC-MS method combined with a chemometric approach to discriminate drug type from fibre type cannabis based on the analysis of the relative proportions of the major compounds found in cannabis seedlings' leaves. Nowadays, this question is of utmost importance for law enforcement authorities in Switzerland, given that persons cultivating cannabis always advocate that the plants are fibre type and then legal. In such situations, the prosecutors ask forensic laboratories to provide a statement about the chemotype of the plant. When the cannabis is mature this problem is solved by measuring the THC concentration from the flowers. However, when laboratories are dealing with seedlings they have to lead the plant to maturity, as required by the EU official cannabis analysis protocol [12], resulting in a time consuming and costly procedure.

\section{Materials and methods}

\subsection{Sampling and growth conditions}

Seeds from 11 Cannabis varieties approved by the Swiss Federal Office for Agriculture [13] were purchased from the Fédération Nationale des Producteurs de Chanvre (Le Mans, France) (fibre type, chemotype III). Seeds from 13 illegal ones were obtained in particular websites (drug type; chemotype I). Cannabis varieties for each chemotype are listed in Table 2 .

In order to determine the chemical profile of each seedling, 15 plants of each variety were greenhouse grown under the following environmental conditions: the temperature varied between $20^{\circ} \mathrm{C}$ and $22^{\circ} \mathrm{C}$ during the growth of the plants, relative humidity was fixed to $50 \%$ automatically and the light/dark photoperiod was hold for 15 and 9 h, respectively, using artificial lighting when necessary.
After 28 days of growth, leafs were picked up from each plant of each variety. Leaves were dried at room temperature and powdered. Three samples by plant ( $100 \mathrm{mg}$ each) were individually extracted with $5 \mathrm{~mL}$ of hexane containing $35 \mathrm{mg}$ squalane per $100 \mathrm{~mL}$ as internal standard (IS). The tube containing the sample and extraction solution was placed in an ultrasonic water bath for $15 \mathrm{~min}$ and then in a rotating beater for $60 \mathrm{~min}$. After filtration, $1 \mathrm{~mL}$ of the solution is then transferred into a vial. Each sample is then individually analysed by GC-MS to separate and to identify the compounds.

\subsection{Analytical methods}

An Agilent 7890A gas chromatograph interfaced with an Agilent 5975C mass selective detector was used for the analysis. Separation was accomplished on a HP$5 \mathrm{~ms}$ capillary column ( $30 \mathrm{~m}$ length, $0.25 \mathrm{~mm}$ i.d. and $0.25 \mu \mathrm{m}$ film thickness). Injections were carried out in split mode using a general purpose split/splitless liner packed with glass wool (Agilent Technologies No. 5183-4711). The temperature program starts at $100^{\circ} \mathrm{C}$, increases to $260^{\circ} \mathrm{C}$ (at $10^{\circ} \mathrm{C} / \mathrm{min}$ ) and holds for $10 \mathrm{~min}$ for a total run of $26 \mathrm{~min} .2 \mu \mathrm{L}$ of each sample was injected with helium as carrier gas (constant flow mode, $1 \mathrm{~mL} / \mathrm{min}$ ) using a split ratio of $1: 20$. Temperatures applied are $280^{\circ} \mathrm{C}$ for injector, $250^{\circ} \mathrm{C}$ for transfer line, $230^{\circ} \mathrm{C}$ for ion source and $150{ }^{\circ} \mathrm{C}$ for quadrupole. Electron multiplier voltage was fixed to $1976 \mathrm{~V}$. Data were acquired in the full scan mode (30-450 m/z mass range) with a sampling rate of 3 ( $1.77 \mathrm{scans} / \mathrm{s})$ and were analysed using MSD Enhanced ChemStation v. D.02.00.275 (Agilent Technologies).

Compounds found in samples were identified using both mass spectrum computerized databases, such as WILEY and NIST05, and data coming from literature $[14,15]$.

\subsection{Chemometrics tools}

\subsubsection{Software}

Data processing was performed using Microsoft Excel 2003 and R version 2.9.0 in which Support Vector Machines (SVMs) classification was performed using the package e1071.

\subsubsection{Data processing}

Areas of targets ions in the chromatograms were integrated for each identified compound. Peaks areas were normalized to the internal standard and the square root was performed in order to reduce the influence of larger peaks and thus to have the variables on a comparable scale. Then, data are scaled to zero mean and unit variance [16].

\subsubsection{Classification methods: $L D A$ and SVMs}

The objective of the methodology is to classify a cannabis sample into drug or fibre type Cannabis. As these two classes could be linearly or non-linearly separable, two classification methods respectively managing these questions have been tested. The potential of linear discriminant analysis (LDA) and SVMs to predict the belonging of a plant to chemotype I (drug type) or chemotype III (fibre type) has been then evaluated. These two methods try to find optimal boundaries between classes in order to discriminate them. LDA produces a linear decision boundary between two classes while SVMs models can be used for complex non-linear situations using an appropriate kernel function. The aim of the SVMs is to find the unique separating hyperplane having the maximum margin of separation between the two classes. A peculiar characteristic of SVMs is that datapoints which lie on the

Table 2

Cannabis varieties used for chemotype determination of drug type and fibre type Cannabis [13].

\begin{tabular}{|c|c|}
\hline Chemotype & Varieties \\
\hline I: 13 drug type cannabis varieties & $\begin{array}{l}\text { Afghan (A), AK47 (AK47), bubble gum (BG), chronic (CH), fraise sativa (FS), granflora (G), ice (I), maple leaf (ML), } \\
\text { monster bloom (MB), northern light (NL), red diesel (RD), skyflight (S) and top } 44(T 44)\end{array}$ \\
\hline III: 11 fibre type cannabis varieties & $\begin{array}{l}\text { Beniko (BEN), epsilon } 68 \text { (EPS), fasamo (FAS), fedora } 17 \text { (FED 17), felina } 32 \text { (FEL), férimon (FER), futura } 75 \text { (FUT), } \\
\text { lovrin } 110 \text { (LOV), kompolti (KOM), uniko B (UB) and USO31 (USO31) }\end{array}$ \\
\hline
\end{tabular}


margins - named support vectors - determine the solution of the classification problem [17]. The best kernel is selected after experimentation. This step is necessary as no theoretical tools exist to determine which kernel performs the best for a given dataset. In our case, the radial basis function (RBF) kernel was an excellent alternative due to his only one hyperparameter to optimize [18], $\gamma$, leading to a reasonable complexity of the SVMs model as pointed out in the RBF equation:

$K\left(x_{i}, x_{j}\right)=\exp \left(-\gamma\left\|x_{i}-x_{j}\right\|^{2}\right)$

with $\gamma>0$ the parameter which determines the width of the RBF and $x_{i}, x_{j}$ the vectors of the $i$ th and $j$ th training samples. As explained in [17], the complexity of the SVMs model can be controlled with an additional parameter, $C$. This penalty parameter controls the trade-off between allowing training errors (low value of $C$ leads to a simpler prediction function) and looking for a more accurate model (high value of $C$ leads to a complex prediction function). $C$ can be adjusted by the user or automatically optimized using some SVMs packages. For a given dataset, $\gamma$ and $C$ have to be optimized in order to obtain best performance results. Detailed explanation can be found in [17-20] for SVMs and [21,22] for LDA methods.

\subsubsection{Optimisation}

While there is no requirement to optimize LDA model, it is necessary for SVMs [23]. In order to find the most performing SVMs model we have determined the optimal $\gamma$ and $C$ values with the function tune.svm in $R$ (library e1071). This function carries out a grid search over specified ranges of $\gamma$ and $C$. The values ranges tested were $0.001-100$ for $\gamma$ and $0.1-1000$ for $C$. The best performance is computed using a 10 -fold cross validation on the whole dataset. The parameter combination with the best performance is chosen and then a suitable SVMs model can be trained using these optimal parameters [20].

\subsubsection{Assessing the model quality}

To assess the quality of the model, cannabis samples were divided into two groups: the training samples on which the model is built, and the test samples on which the predictive capability is tested. The class membership is known for the two groups. The classification rate for each model is then calculated by measuring the percentages of samples correctly classified for training and test samples. The percentage of samples incorrectly classified for each class was also evaluated. They have been named as false positive drug (FPD) and false positive fibre (FPF). False positive drug is defined as the percentage of samples classified as drug type cannabis whereas they are fibre type cannabis. False positive fibre is defined as the percentage of samples classified as fibre type cannabis whereas they are drug type cannabis. To ensure that the results are well representative of the model classification ability and do not depend on the samples used, the separation into training and test sets is repeated 1000 times. Samples are randomly chosen each time. Classification measures are recorded over all 1000 iterations and the reported values are mean values (cf. Fig. 1). Finally, after the creation of the model a validation test is performed with an independent validation set. The latter consists of 44 cannabis seedlings coming from police seizures where growing conditions are
DEVELOPMENT OF CLASSIFICATION MODELS

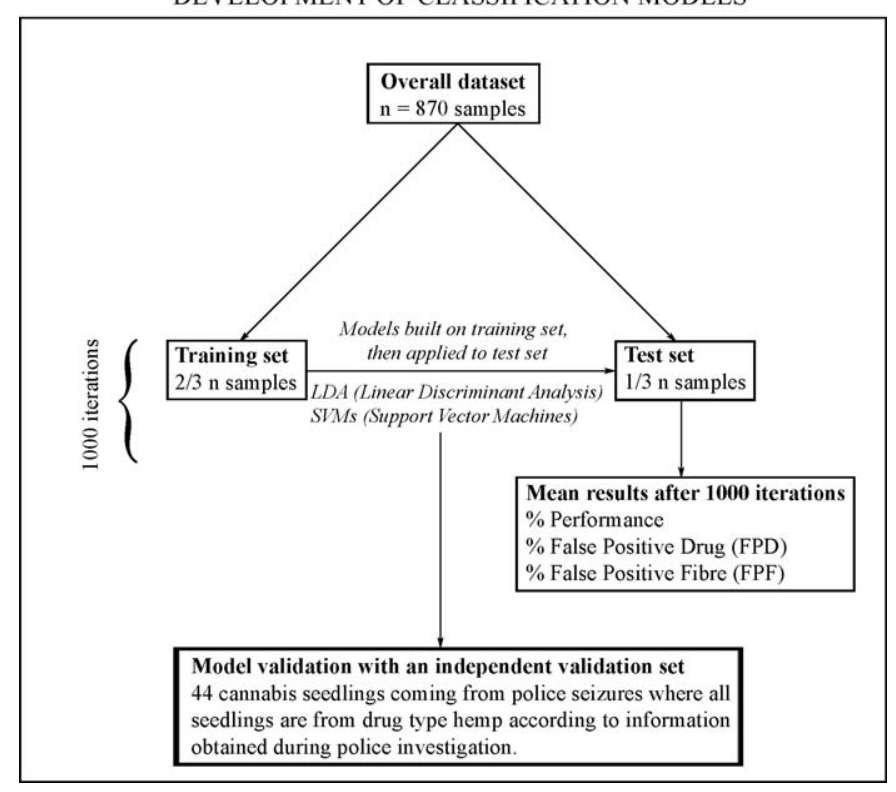

Fig. 1. Statistical methodology applied during this work.

not known but likely different from ours. All seedlings are from drug type cannabis according to information obtained during police investigation. Indeed, Cannabis seedlings were labelled by raisers using the name of their variety. Furthermore, defendants made a full confession and listed all Cannabis varieties that they cultivated.

\section{Results and discussion}

In this study, we have focused our attention on the overall set of compounds presents at detectable amounts in the leaves of cannabis seedlings. Fifteen compounds have been selected taking into account their presence in drug and fibre type seedlings cannabis. Fig. 2 shows a typical chromatogram obtained for a cannabis seedling analysis. Table 3 shows compounds retention times (relative to squalane) as well as their specific target ions and qualifiers.

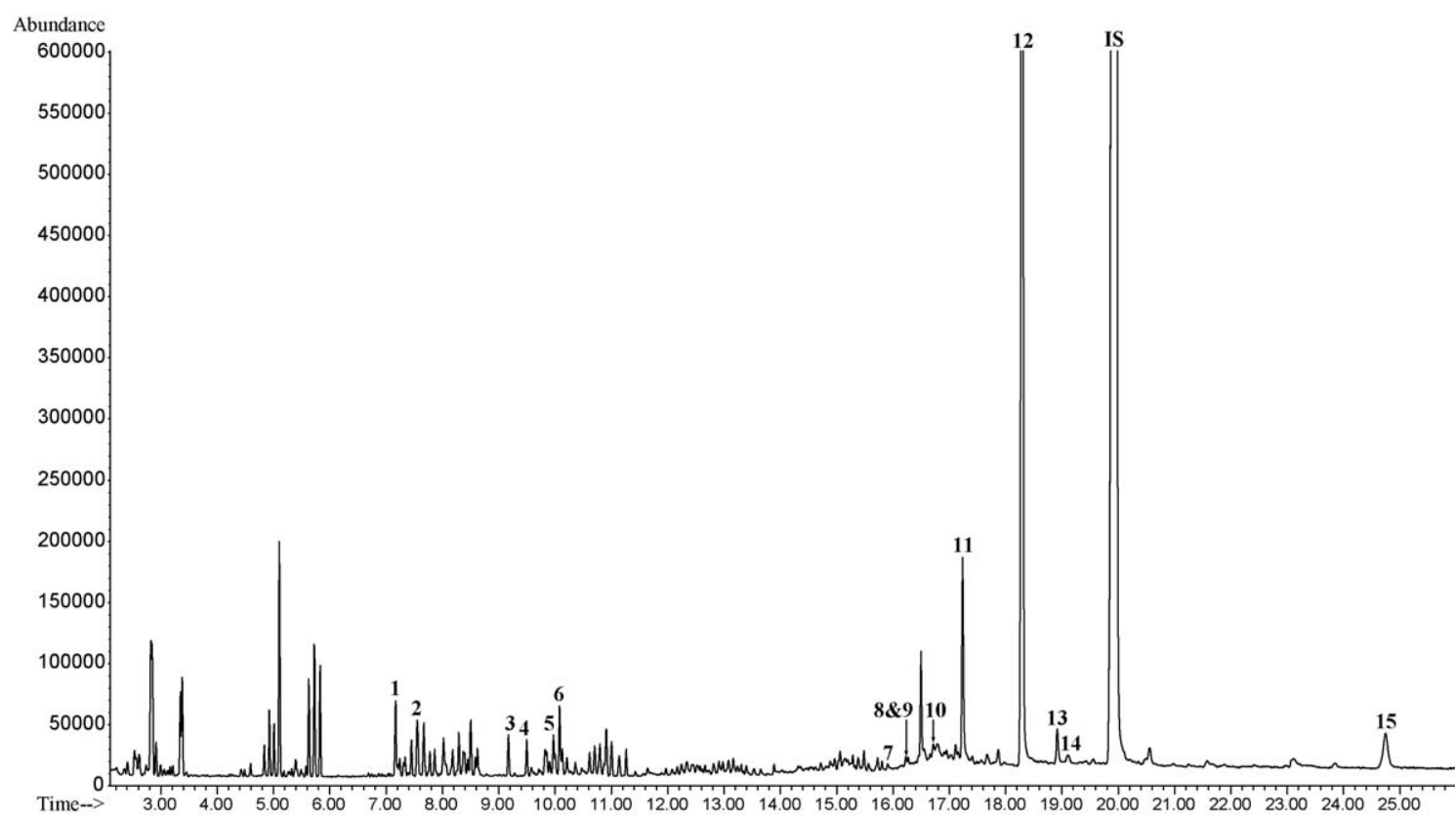

Fig. 2. Total ion current (TIC) chromatogram of a typical drug type cannabis sample. Number on the chromatogram refers to Table 3. 
Table 3

Compounds found in leaves, their relative retention time (RRT), target ions and qualifiers.

\begin{tabular}{|c|c|c|c|}
\hline $\begin{array}{l}\text { Peak } \\
\text { numbers }\end{array}$ & Compounds & $\begin{array}{l}\text { RRT } \\
(\mathrm{min})\end{array}$ & $\begin{array}{l}\text { Target ion, } \\
\text { qualifiers }(\mathrm{m} / \mathrm{z})\end{array}$ \\
\hline 1 & Beta caryophyllene & 0.360 & 93, $133,41,69$ \\
\hline 2 & Alpha caryophyllene & 0.379 & $\mathbf{9 3}, 80,121,147$ \\
\hline 3 & Guaiol & 0.460 & $\mathbf{1 6 1}, 107,59,93$ \\
\hline 4 & $\gamma$-Eudesmol & 0.476 & 189, $161,204,133$ \\
\hline 5 & Bulnesol & 0.500 & 107, 135, 93, 161 \\
\hline 6 & $\alpha$-Bisabolol & 0.506 & $109,119,69,43$ \\
\hline 7 & Cannabinoid 1 & 0.797 & 231, 314, 299, 271 \\
\hline 8 & $\begin{array}{l}\text { Tetrahydrocannabivarin } \\
\text { (THV) }\end{array}$ & 0.813 & 271, 286, 203, 243 \\
\hline 9 & Cannabinoid 2 & 0.823 & 231, 314, 174, 243 \\
\hline 10 & Cannabicyclol (CBL) & 0.839 & $\mathbf{2 3 1}, 232,174,314$ \\
\hline 11 & Cannabidiol (CBD) & 0.864 & 231, 174, 314, 299 \\
\hline 12 & $\Delta^{9}-\mathrm{THC}(\mathrm{THC})$ & 0.918 & 299, 314, 231, 271 \\
\hline 13 & Cannabigerol (CBG) & 0.949 & $193,231,123,316$ \\
\hline 14 & Cannabinol (CBN) & 0.959 & $\mathbf{2 9 5}, 238,310,223$ \\
\hline 15 & Nonacosane & 1.241 & $57,71,85,43$ \\
\hline
\end{tabular}

In this work discrimination between fibre type and drug type cannabis using $([\mathrm{THC}]+[\mathrm{CBN}]) /[\mathrm{CBD}]$ ratio leads to the misclassification of fibre type (0.6\% of fibre type samples misclassified) as well as drug type cannabis (7.5\% of drug type samples misclassified) as illustrated in Fig. 3. Drug type samples which are misclassified are coming in the majority from one variety.

Therefore, it is necessary to select compounds allowing a better differentiation between fibre type and drug type cannabis. Principal component analysis (PCA) and boxplots were then performed [21]. Eight compounds having high discrimination capabilities have been selected: they are guaiol, $\gamma$-eudesmol, bulnesol, $\alpha$-bisabolol, THV, CBD, THC and CBN. With these compounds a PCA has been performed in order to visualize the representation of each sample using the scores plot against the first principal component (PC1) and second principal component (PC2). Fig. 4 illustrates the scores of cannabis samples against the first two principal components. Some fibre type and drug type cannabis samples are non-linearly separable leading to an overlapping area (circle in Fig. 4). Results for the classification performed with LDA and SVMs models are shown in Table 4. The high FPF rate observed for LDA model highlights the difficulty to separate cannabis samples with a linear classifier. In order to decrease this value it has been decided to use a non-linear algorithm that is implemented with the SVMs model. SVMs performed much better than LDA with a classification performance above 99\% over 1000 iterations. Furthermore, as expected, the FPF rate has dramatically decreased. SVMs used 122 support vectors to create the classification model, 95 in drug type samples and 27 in fibre type samples. The hyperparameters for RBF kernel were 1 for $\gamma$ and 100 for $C$.

The main idea of the model was to maximise the classification of fibre type cannabis (minimise the false positive drug rate), in such a way that a person who legally grow Cannabis would not be judicially prosecuted by error. The low false positive rates observed are in agreement with this concept. As plants have been cultivated in the same environmental conditions, variations in cannabis samples' composition are mainly due to genetics characteristics (the seed). These variations have been well evaluated by the SVMs model given that discrimination between fibre type and drug type cannabis has been observed using selected compounds.

Furthermore, $100 \%$ of the validation set has been correctly classified and thus samples predicted as drug type cannabis by the SVMs model. As far as LDA, about $11 \%$ of the samples were erroneously classified as fibre type cannabis. SVMs model's ability to correctly classify the validation set demonstrates that the model can handle the case when new samples have grown under different environmental conditions. Therefore genetics characteristics are more important factors than environmental ones. Moreover, it confirms that the SVMs classification power is better than the linear algorithm one.

As presented in a recent study the chemotype does not change in plants at different ages or in different sexes throughout the entire life cycle until flowering [8,24]. Thus, the SVMs model that has been built can be used to discriminate between fibre and drug type cannabis at an early stage of growth. Consequently, it is not necessary to cultivate during several months seized seedlings to sample their flowering portions and to quantify the amount of THC. This actual procedure is time consuming (time of growth) and expensive (cost of the standard used for quantification, cost of a secured greenhouse and cost of materials required for culture). For chemotype identification, sampling leaves at an early stage of development and the determination of the relative amount of guaiol, $\gamma$-eudesmol, bulnesol, $\alpha$-bisabolol, THV, CBD, THC and CBN

Plot of $(\mathrm{THC}+\mathrm{CBN}) / \mathrm{CBD}$ values for each sample

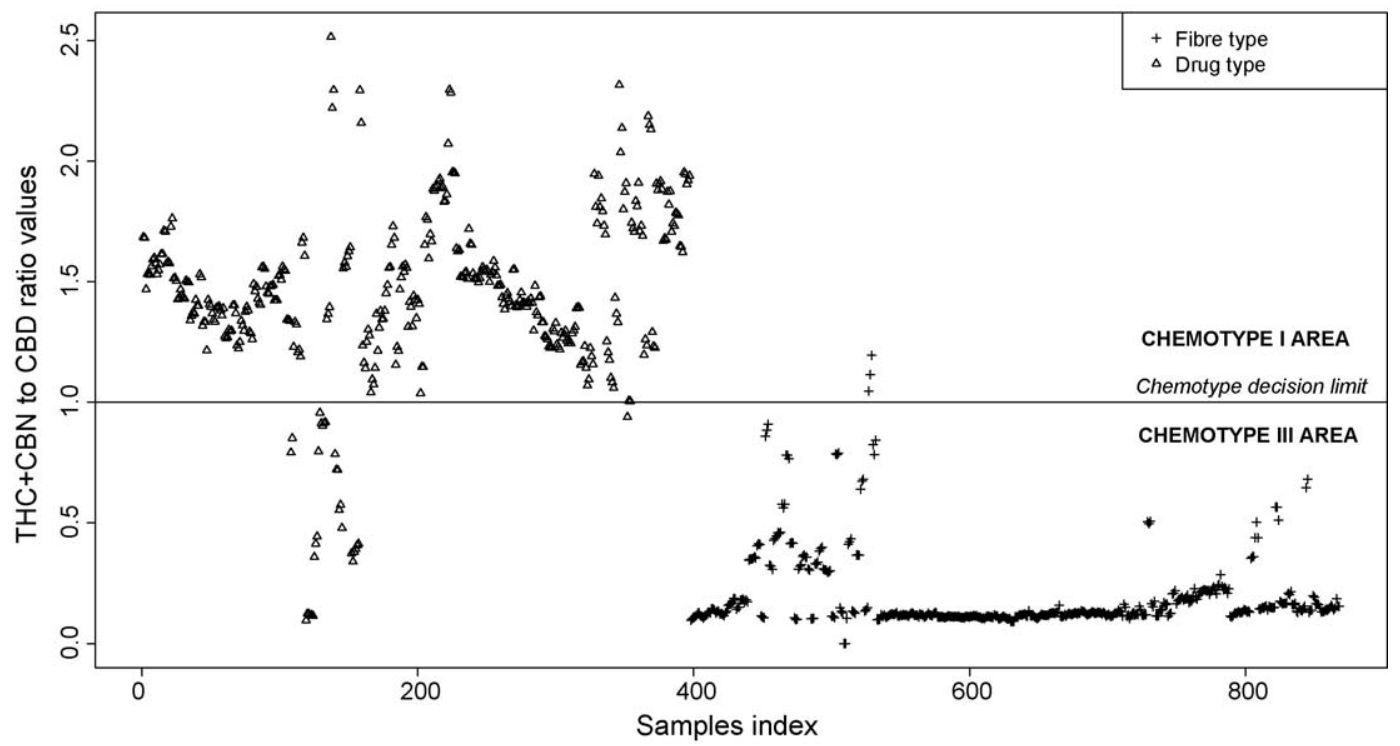

Fig. 3. Plot of $\Delta^{9}$-tetrahydrocannabinol (THC) + cannabinol (CBN) to cannabidiol (CBD) ratio values for each cannabis sample. 
Plot of cannabis samples' scores on PC1 and PC2

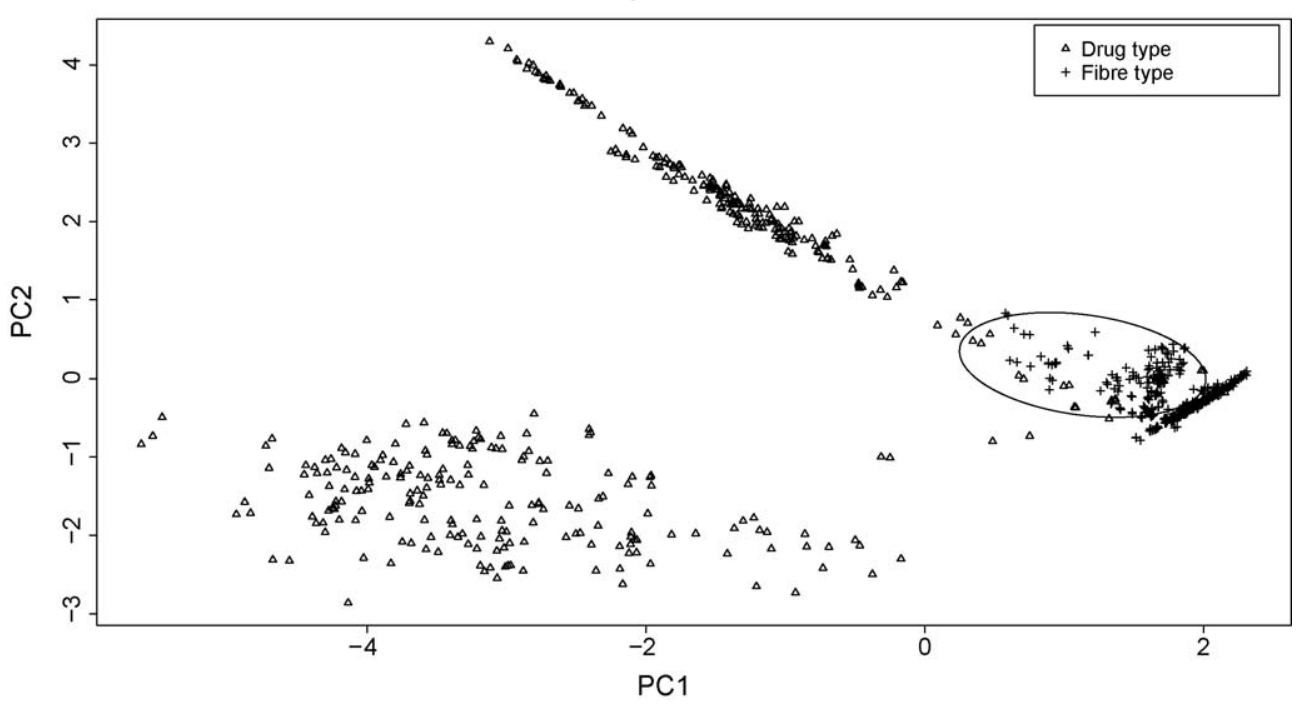

Fig. 4. Plot of cannabis samples scores against the first principal component (PC1) and second principal component (PC2).

Table 4

Results for LDA and SVMs showing mean values of the classification measures over 1000 iterations with the standard deviation in brackets.

\begin{tabular}{|c|c|c|c|c|c|c|}
\hline & \multicolumn{3}{|l|}{ Training set } & \multicolumn{3}{|l|}{ Test set } \\
\hline & \%Performance & \%FPD & $\% \mathrm{FPF}$ & \%Performance & \%FPD & \%FPF \\
\hline LDA & $97.3( \pm 0.4)$ & $0.3( \pm 0.2)$ & $5.5( \pm 0.9)$ & $97.0( \pm 1.0)$ & $0.3( \pm 0.5)$ & $6.0( \pm 2.3)$ \\
\hline SVMs & $99.7( \pm 0.1)$ & 0.0 & $0.7( \pm 0.3)$ & $99.2( \pm 0.7)$ & $0.3( \pm 0.8)$ & $1.3( \pm 1.2)$ \\
\hline
\end{tabular}

would be a potential modification of the EU official cannabis analysis protocol. The present method could be applied to the production control of non-scheduled (fibre type) and scheduled (drug type) Cannabis. In addition, an option to confirm the results obtained by the SVMs model is the quantification of THC once the seedling has reached the stage of maturity and florescence.

\section{Conclusions}

In this work an original Cannabis classification model has been developed allowing the discrimination between fibre- and drug type Cannabis seedlings by GC/MS based on non-cannabinoid and cannabinoid leaf constituents. Using selected compounds the SVMs model correctly classifies samples with a classification performance above $99 \%$ and with low false positive rates (less than $2 \%$ ). The GC-MS method is consequently valid, fast and cheap regarding the classification of a cannabis seedling in comparison to the EU official procedure, i.e. cultivation of seedlings to mature plants. Thus, it is not necessary to wait for the plant's florescence, to sample their flowering portions and quantify the amount of THC - the actual time consuming and expensive procedure - in order to determine their chemotype. The SVMs model that has been built can be used to discriminate between fibre and drug type cannabis at an early stage of growth ( 1 month of growth in this work).

Chemotype identification by sampling leaves at an early stage of development and determination of the relative amount of guaiol, $\gamma$-eudesmol, bulnesol, $\alpha$-bisabolol, THV, CBD, THC and CBN is a valuable tool that could be included in EU official cannabis analysis protocol.

\section{Acknowledgments}

The authors would like to thank Daniel Alvarez, Cédric Liengme, Kevin Rudaz and Blaise Tissot-Dit-Sanfin from the département de biologie moléculaire végétale of the University of Lausanne for the technical support.

\section{References}

[1] C.E. Turner, M.A. Elsohly, E.G. Boeren, Constituents of Cannabis-Sativa L. 17. A review of the natural constituents, Journal of Natural Products 43 (2)(1980) 169234.

[2] F.E. Dussy, C. Hamberg, M. Luginbühl, T. Schwerzmann, T.A. Briellmann, Isolation of [Delta]9-THCA-A from hemp and analytical aspects concerning the determination of [Delta]9-THC in cannabis products, Forensic Science International 149 (1) (2005) 3-10.

[3] R. Brenneisen, Chemistry and analysis of phytocannabinoids and other cannabis constituents, in: M.A. Elsohly (Ed.), Marijuana and the Cannabinoids, Humana Press, Totowa, NY, 2007, pp. 17-49.

[4] M.A. ElSohly, D. Slade, Chemical constituents of marijuana: the complex mixture of natural cannabinoids, Life Sciences 78 (5) (2005) 539-548.

[5] E. Small, H. Beckstead, Common cannabinoid phenotypes in 350 stocks of Cannabis, Lloydia-The Journal of Natural Products 36 (2) (1973) 144-165.

[6] United Nations Office on Drugs and Crime (UNODC), Recommended Methods for the Identification and Analysis of Cannabis and Cannabis Products, (2009) http:// www.unodc.org/documents/scientific/ST-NAR-40-Ebook.pdf (retrieved October 2009).

[7] M. Stefanidou, A. Dona, S. Athanaselis, I. Papoutsis, A. Koutselinis, The cannabinoid content of marihuana samples seized in Greece and its forensic application, Forensic Science International 95 (2) (1998) 153-162.

[8] D. Pacifico, F. Miselli, A. Carboni, A. Moschella, G. Mandolino, Time course of cannabinoid accumulation and chemotype development during the growth of Cannabis sativa L., Euphytica 160 (2) (2008) 231-240.

[9] A.F. Vogelmann, J.C. Turner, P.G. Mahlberg, Cannabinoid composition in seedlings compared to adult plants of Cannabis-Sativa, Journal of Natural Products 51 (6) (1988) 1075-1079.

[10] Official Journal of the European Communities of 29 February 2000 (C 56 E/18), Commission Regulation (EC) modifying regulation EC n $1251 / 1999$, http://eurlex.europa.eu/LexUriServ/LexUriServ.do?uri=OJ:C:2000:056E:0017:0018:FR:PDF (retrieved October 2009).

[11] Ordonnance du Département Fédéral de l'Economie sur les semences et les plants des espèces de grandes cultures et de plantes fourragères, p. 59, http://www.ad$\mathrm{min} . \mathrm{ch} / \mathrm{ch} / \mathrm{f} / \mathrm{rs} / 9 / 916.151 .1$.fr.pdf (retrieved October 2009).

[12] Official Journal of the European Communities of 21 February 2002 (L 51/21) Commission Regulation (EC) No. 327/2002, http://eur-lex.europa.eu/LexUriServ/LexUriServ.do?uri=OJ:L:2002:051:0014:0022:FR:PDF (retrieved October 2009). 
[13] Ordonnance de l'Office Fédéral de l'Agriculture sur le catalogue des variétés de céréales, de pommes de terre, de plantes fourragères, de plantes oléagineuses et à fibres ainsi que de betteraves, RS 916.151.6, http://www.admin.ch/ch/f/as/2009/ 2441.pdf (retrieved October 2009).

[14] A. Hazekamp, A. Peltenburg, R. Verpoorte, C. Giroud, Chromatographic and spectroscopic data of cannabinoids from Cannabis sativa L., Journal of Liquid Chromatography \& Related Technologies 28 (15) (2005) 2361-2382.

[15] R. Lewis, S. Ward, R. Johnson, D.T. Burns, Distribution of the principal cannabinoids within bars of compressed cannabis resin, Analytica Chimica Acta $538(1 / 2)$ (2005) 399-405.

[16] C. Reimann, P. Filzmoser, R. Garrett, R. Dutter, Statistical Data Analysis Explained: Applied Environmental Statistics with R, John Wiley \& Sons, Chichester, England/ Hoboken, NJ, 2008.

[17] O. Ivanciuc, Applications of support vector machines in chemistry, in: K.B. Lipkowitz, T.R. Cundari (Eds.), Reviews in Computational Chemistry, vol. 23, Wiley-VCH, Weinheim, 2007, pp. 291-400.

[18] C.W. Hsu, C.C. Chang, C.J. Lin, A Practical Guide to Support Vector Classification, Department of Computer Science and Information Engineering, National Taiwan University, 2003.
[19] H.D. Li, Y.Z. Liang, Q.S. Xu, Support vector machines and its applications in chemistry, Chemometrics and Intelligent Laboratory Systems 95 (2) (2009) 188-198.

[20] A. Karatzoglou, D. Meyer, K. Hornik, Support vector machines in R, Journal of Statistical Software 15 (9) (2006).

[21] D.L. Massart, B.G.M. Vandeginste, L.M.C. Buydens, S. De Jong, P.J. Lewi, J. SmeyersVerbeke, Handbook of Chemometrics and Qualimetrics: Part A, Elsevier, Amsterdam, 1997.

[22] B.G.M. Vandeginste, D.L. Massart, L.M.C. Buydens, S. De Jong, P.J. Lewi, J. SmeyersVerbeke, Handbook of Chemometrics and Qualimetrics: Part B, Elsevier, Amsterdam, 1998.

[23] S.J. Dixon, R.G. Brereton, Comparison of performance of five common classifiers represented as boundary methods: Euclidean Distance to Centroids, Linear Discriminant Analysis, Quadratic Discriminant Analysis, Learning Vector Quantization and Support Vector Machines, as dependent on data structure, Chemometrics and Intelligent Laboratory Systems 95 (1) (2009) 1-17.

[24] G. Fournier, J. Bausset, O. Beherec, M. Desvals, S. Bertucelli, The simplification of the industrial hemp crops control is possible, Annales de Toxicologie Analytique 19 (3) (2007) 201-209. 